\title{
Tick-borne Relapsing Fever in Children in the North-west of Iran, Qazvin
}

\author{
Parviz Ayazi ${ }^{1}$,Abolfazl Mahyar ${ }^{2}$, Sonia Oveisi ${ }^{3}$, \\ Neda Esmailzadehha ${ }^{3}$, Sadralnesa Nooroozi ${ }^{2}$ \\ ${ }^{1}$ Department of Pediatric Infectious Diseases, Qazvin University of Medical \\ Sciences, Qazvin, Iran; \\ ${ }^{2}$ Department of Pediatrics, Qazvin University of Medical Sciences, Qazvin, Iran; \\ ${ }^{3}$ Metabolic Diseases Research Center, Qazvin University of Medical Sciences, \\ Qazvin, Iran
}

Received December 10, 2014; Accepted September 15, 2015.

Key words: Borrelia - Tick-borne relapsing fever (TBRF) - Children - Clinical Laboratory

\begin{abstract}
Relapsing fever is caused by the Borrelia species of spirochetes. Louseborne epidemics of the disease may happen but the endemic disease is generally transmitted to humans by the bite of an infected tick (Ornithodorus). Clinical and laboratory findings of tick-borne relapsing fever in children in the north-west of Iran, Qazvin, were evaluated. This study was conducted from September 1992 to September 2012. Records from 53 cases of tick-borne relapsing fever (TBRF) were reviewed. In positive cases, febrile illness, and spirochetes were recognized in peripheral blood preparations. Of the 53 children younger than 12 years, fifty two percent were male and about one third (34\%) of the patients were in the age range of 7-12 years. The disease is recorded through the whole year but its peak occurs during summer (52.8\%) and autumn (32.1\%). Sixty eight percent of patients were living in urban areas but had frequent travel to rural area. Thirty two percent of the cases were living in rural areas where their dwellings were close to animal shelters. All (100\%) of the 53 subjects were febrile. Travellers to the rural areas with high prevalence of the disease should be attentive of the risk of tick-borne relapsing fever and use suitable control measures. Consequently relapsing fever should be considered when patients who live in or have vacationed in north-west of Iran show a recurring febrile illness.
\end{abstract}

Mailing Address: Assoc. Prof. Parviz Ayazi, Department of Pediatric Infectious Diseases, Qazvin Children Hospital, Shahid Beheshti Blvd., Qazvin, Iran; Phone: +98 91218237 97; Fax: +98 28333440 88; e-mail: parviz_ayazi@yahoo.com 


\section{Introduction}

The tick-borne relapsing fever (TBRF) is an acute arthropod-borne infectious illness found in the Middle East, Africa, Europe and North and South America. Of the significant clinical manifestation of the disease are the returning attacks of fever and chill. The etiology of the disease is numerous species of Borrelia which are transmitted by species of soft ticks (Mahram and Ghavami, 2009). All species which are pathogenic to man are transmitted by ticks of the genus Ornithodoros but Borrelia recurrentis is the only one which is transmitted by lice (Sonenshine, 1997; Raoult and Roux, 1999). Relapsing fever is caused by spirochetes through the contact by infected lice (Pediculus humanus) or the bite of an infected tick (Ornithodoros species). The range of incubation period is 4 to 18 days and even in some study more than 18 days (mean 7 days) (Southern and Sanford, 1969).

Louse-borne relapsing fever (LBRF or epidemic relapsing fever) is causes by Borrelia recurrentis. The insect's infected hemolymph which contains spirochetes is released and contaminates abraded or normal skin and mucous membranes by crushing the louse (e.g. when scratching). Locations with reduced sanitation and disastrous events are an ideal place for LBRF propagation through the community. About 5 million deaths following LBRF and around 15 million patients with final diagnosis of LBRF in Africa, Eastern Europe, and Russia make an outstanding feature of this disease in the world in this century (Goubau, 1984).

Tick-borne relapsing fever (TBRF) or endemic relapsing fever is found throughout most of the world, and it is not specific to a particular region. TBRF is found throughout most of the western United States, southern British Columbia, the plateau regions of Mexico and Central and South America, the Mediterranean, Central Asia, and it is endemic in the Africa. Number of patients in different countries varies, for example in Israel it is eight cases per year comparing to Iran which is 72 cases per year (Assous and Wilamowski, 2009). The tick vectors, Ornithodoros species, are argasid (soft) ticks with painless bite and feeding mainly during the night. Rodents are the primary reservoirs of Borrelia (Goubau, 1984). Ornithodoros ticks may live long between stings and carry spirochetes for prolonged periods; in contrast the body louse which lives only a few weeks (Rebaudet and Barola, 2006).

Between Borrelia species, the vector ticks and the distribution area a particular associations generally exist (Rebaudet and Barola, 2006). Ornithodoros tholozani is recognized as the major vector distributed in the Middle East and it is the only reservoir for Borrelia persica, which is often accountable for old-world TBRF. Species of these ticks live in proximity of humans and feeding occurs throughout the night for short periods, by a painless bite (Barbour and Hayes, 1986). One of the important sanitation troubles in Middle East and Central Asia is tick-borne relapsing fever.TBRF is endemic in the north-west of the Islamic Republic of Iran and relatively high prevalence of disease in the mentioned country resulted in many clinical and epidemiological researches (Arshi et al., 2002; Ghavami et al., 2002). 
Tick, Borrelia species as well as ecological characteristic in every part of the world and especially in each province play major role in epidemiology of the disease. Reservoir hosts are generally wild rodents. Tick gets infection during feeding on an infected vertebrate and this is the usual way of tick infection. Transition of the pathogen to one more vertebrate is carried out during tick feeding in the next life period (Barmaki et al., 2010). The major manifestation of the disease is the recurrence of fever, which at the time of fever peak is accompanied with high bacteraemia (Southern and Sanford, 1969; Felsenfeld, 1971; Cook and Zumla, 2002). The mutual action among host antibody response and bacterial antigenic variation of external proteins causes recurrence characteristic of the disease (Barbour, 1989; Dai et al., 2006). The most commonly used methods for laboratory and final diagnosis of tick-borne relapsing fever is thin and thick blood smears, with a sensitivity of $80 \%$ (Assous and Wilamowski, 2009). TBRF has a wide range of mortality $(2-40 \%)$ and children have the highest mortality. Relapsing fever also causes complications during pregnancy such as stillbirth (Southern and Sanford, 1969). The purpose of this study was to evaluate clinical and laboratory findings of tick-borne relapsing fever in children of the north-west of Iran, Qazvin.

\section{Material and Methods}

Qazvin Children Hospital provides tertiary health care for Qazvin province and is a referral hospital for the surrounding area. Qazvin province covers $15,821 \mathrm{~km}^{2}$ between 48-45 to 50-50 east of Greenwich Meridian of longitude and 35-37 to 36-45 north latitude of the equator. Peripheral blood smear are examined regularly for Borrelia spirochetes from most patients presenting to the Department of Pediatric Infectious Diseases of Qazvin Children Hospital with fever or a history of fever and especially when travelled to the rural area. Between September 1992 to September 2012, data were collected from 53 hospital medical record of patients with ultimate diagnosis of relapsing fever and all of them had peripheral smear containing Borrelia.

Children younger than 12 years were included in the present study. The following information was collected. From the epidemiological point of view, the items such as name, age, sex, rural or urban, season were recorded.

Concerning clinical features, the following such as fever, chill, headache, nausea, vomiting, sweating, abdominal pain, and cough were included. Regarding physical examination the following finding were included, temperature, the presence of jaundice or meningism, and spleen, liver and lymphnode size. At top of paraclinical studies the most outstanding finding included spirochetes in the peripheral blood smears stained with Gimsa stain. In addition erythrocyte sedimentation rate (ESR), qualitative C-reactive protein (CRP), complete blood count especially white blood cells $(\mathrm{WBC})$ and platelet, were included in the study.

Most patients were treated with erythromycin (69.8\%), daily for 5 days. Ten patients were treated with ceftriaxon, five patients with penicillin and one with 
ceftizoxim. Observations for side effect of drugs such as Jarisch-Herxheimer reaction were included in the study (Barclay and Coulter, 1990).

\section{Results}

Fifty three children with diagnosis of TBRF were hospitalized in the hospital during twenty years. Of the 53 children younger than 12 years, fifty two percent were male and about one third (34\%) of the patients were in the age range of 7-12 years. Since all the patients in this study were children, they were not employed outside the home and were not working. About $68 \%$ were living in urban area but with frequent travel to rural area. $32 \%$ of the patients were living in the countryside where their dwellings were close to animal shelters. The disease is recorded through the whole year but its peak occurred during summer $(52.8 \%)$ and autumn (32.1\%). All (100\%) of the 53 subjects were febrile, so the most common clinical sign was fever. About 31 patients (58.5\%) had fever between 38 to $39^{\circ} \mathrm{C}$ and 22 cases (41.5\%) had a fever over $39^{\circ} \mathrm{C}$.

Vomiting in 26 patients (49.1\%), headache in 21 (39.6\%), weakness and lethargy in $18(34 \%)$ and abdominal pain in 16 patients (30.2\%),) was reported. Chills in 14 patients $(26.5 \%)$, cough in $11(20.8 \%)$, myalgia in $11(20.8 \%)$, sweating in $9(17 \%)$ and seizure in 6 patients $(11.3 \%)$ was observed.

Hepatomegaly on palpation in 15 patients (28.3\%), abdominal tenderness in 10 (18.9\%) and lymphadenopathy in 3 patients (5.7\%), existed.

All patients had positive spirochete peripheral blood smear. Leukocytosis was found in 43 patients (81.1\%), but in 9 patients (17\%) were normal and only in one case $(1.9 \%)$ decrease in leukocytes was reported. Erythrocyte sedimentation rate (ESR) was between $10 \mathrm{~mm} / \mathrm{h}$ and $100 \mathrm{~mm} / \mathrm{h}$ in 33 cases (62.2\%), and 10 patients (18.9\%) had elevated ESR above $100 \mathrm{~mm} / \mathrm{h}$ and in 10 patients (18.9\%) it was in normal range. Positive CRP was found in all patients. Lumbar puncture was carried out in 19 patients (35.8\%) and all the cases were reported to have been normal.

Erythromycin in 37 patients (69.8\%) was the most commonly used antibiotic. Ceftriaxone in 10 cases, penicillin in 5, and ceftizoxim was used in 1 case. There was no child mortality due to TBRF in the study subjects.

\section{Discussion}

The number of TBRF cases is different among countries, for example in Israel it is eight cases per year and on the other hand in Iran it is about 72 patients per year (Assous and Wilamowski, 2009). Although Masoumi Asl et al. (2009) believe and emphasize that in Iran, where the disease is endemic, especially in the provinces that are located in the north-western part of the country and are mountainous, the incidence of TBRF is much higher. They report that even more than 200 cases of TBRF yearly most probably does not show the real incidence and it is an under estimation. Qazvin province is recognized as a district that TBRF is endemic in it since it was reported in Iran. TBRF has since been documented all through of the 
Iran especially in the provinces of northwest of country, including Ardabil, followed by Hamadan, Zanjan, Kurdestan and Qazvin provinces sequentially. Ardabil province has the highest incidence and is highly infected area $(625$ out of 1,415) (Masoumi Asl et al., 2009). Although TBRF is endemic in provinces of the northwest of Iran but its geographic distribution should be considered since the report of an outbreak of TBRF in Iran (Masoumi Asl et al., 2009).

One of the most important finding was closeness of the patient's home who were living in the village to the livestock such as cattle and sheep. These findings are in accordance with studies of Arshi et al. (2002) in Iran and Abidov et al. (1993) in Uzbekistan.

The present study emphasizes geographic distribution of TBRF and demonstrates the extent of disease in Qazvin province. Although TBRF, and sporadically louse-borne relapsing fever (LBRF), are reportable in Qazvin province, this study suggests that TBRF is much more common than LBRF in this province. For calculating the accurate incidence of TBRF we have to improve the diagnosis and reporting mechanism. Peripheral blood smears is the diagnostic standard test for recognition of spirochetes in the blood and finally the accurate diagnosis of TBRF. Although peripheral blood smears is the diagnostic standard test for detection of spirochetes, but three issues may result in poor diagnosis. One of them and the most important one is the laboratory technician which does not have enough experience. The other one is increased use of automated differential count instead of manual differential count as a cost-saving plan. So we strongly recommend manual differential count by an experienced technician especially in the provinces that TBRF is endemic. The third one is evaluation of blood in the asymptomatic period. During this period the numbers of spirochetes is very low and so are undetectable. Even sometimes could not be found totally in the circulation (Dworkin et al., 1998). Some authors recommend when there is flulike symptom with a history of contact especially in endemic district, the manual differential count should be considered. One of the most important problems in diagnosis of TBRF is during afebrile period when numbers of bacteria are very low in the peripheral smear, so even the experienced technician could not detect the spirochetes. There are other techniques that could compensate this insufficiency. Centrifuged-based enrichment technique is one of the techniques that could easily compensate this insufficiency and it is five hundred more sensitive than thick smears. Some authorities emphasize that centrifuged-based enrichment technique is more valuable than some PCR assay. Since centrifuged-based enrichment technique does not need advanced laboratory equipment and its performance is easy, it is strongly recommended for diagnosis TBRF in endemic area especially in rural part (Naddaf et al., 2011).

All of the cases in our study were children, but in Arshi et al. (2002) study the adult cases were also included, although the majority of cases were children, which is similar to the finding of on tick-borne relapsing fever in Tanzania (Barclay 
and Coulter, 1990). In present study $66 \%$ of cases were younger than 7 years old, compared to $33 \%$ of the patients in Arshi et al. (2002) study and $88 \%$ in Barclay and Coulter (1990) study. Arshi emphasize that although differences between the population distribution of Iran and Tanzania can clarify these findings, it does not completely explain the differences. In Barclay and Coulter (1990) study, 36\% of the cases were infants, comparing ours with $15.1 \%$ of patients were infants but no infants in Arshi et al. (2002) study. Other factors that may explain these differences include the type of spirochete, entomologic and genetic factor. In addition underdiagnosis of the TBRF in newborns, different way of infant care, and approach to fever in endemic area by healthcare employees may somehow explain these differences (Melkert and Stel, 1991). Since some articles have reported vertical transmission as a likely method of acquiring the relapsing fever in newborns, so screening of newborn in endemic district especially in mother with a past history of TBRF or a past history compatible with TBRF, is recommended (Brasseur, 1985; Yagupsky and Moses, 1985; Mahram and Ghavami, 2009).

The most common clinical manifestations of relapsing fever in the present study were fever. The disease was frequently characterized by a high fever, nausea, vomiting, and headache. About 41.5 percent of the patients had a recorded temperature of more than $39^{\circ} \mathrm{C}$; other patients had fever less than $39^{\circ} \mathrm{C}$. The clinical signs and symptoms in present study are relatively comparable to the studies that high temperature, vomiting, headache, abdominal pain, chills, cough, sweating, and nausea were remarkable finding (Table 1). Many patients had frequent relapse or at least two relapses. This finding shows the misdiagnosis of TBRF by physicians especially in endemic area that more attention should be paid to TBRF. These outcomes are to some extent consistent with other researches (Dworkin et al., 1998).

Compared to the other studies, there were no petechiae in our study (Table 1) (0\% vs. 2.8\%, 34\%, 43\% and 54\%) (Brown et al., 1988; Borgnolo et al., 1993a, b; Arshi et al., 2002). Some authors believe this difference may be due to the type of relapsing fever; for example, in louse-borne relapsing fever, thrombocytopenia is much more common comparing TBRF. Although none of the patients in this study had thrombocytopenia but other researchers documented thrombocytopenia (Dworkin et al., 1998). For example in Dworkin et al. (1998) study there were five cases with platelet counts of less than $20,000 / \mathrm{mm}^{3}$, but no major bleeding complications were found. On the other hand, hemorrhagic complications are more common in the LBRF (e.g. purpura, hemoptysis, hematemesis, bloody diarrhea, hematuria, subarachnoid and cerebral hemorrhages, splenic rupture, and retinal hemorrhage) (Goubau, 1984). Lower urinary tract infection is the other complication of TBRF and cause of hematuria, which spirochetes could be recognized in urine (Linnemann et al., 1978).

There have been some findings in other studies but in our study there was no such result (e.g. dysuria, proteiuria, microhematuria). In addition in acute TBRF, 


\section{Table 1 - Comparison of clinical manifestations in different studies}

\begin{tabular}{lccccc}
\hline Clinical findings & $\begin{array}{c}\text { This } \\
\text { study }\end{array}$ & $\begin{array}{c}\text { Arshi } \\
\text { et al. } \\
(2002)\end{array}$ & $\begin{array}{c}\text { Borgnolo } \\
\text { et al. } \\
(1993 \mathrm{~b})\end{array}$ & $\begin{array}{c}\text { Borgnolo et al. } \\
(1993 \mathrm{a}) \\
\text { (children) }\end{array}$ & $\begin{array}{c}\text { Brown } \\
\text { et al. } \\
(1988)\end{array}$ \\
\hline fever & 100.0 & 93.3 & 99 & 100.0 & 73 \\
chills & 26.4 & 86.2 & - & 74.0 & $\left(\right.$ T > 39 $\left.{ }^{\circ} \mathrm{C}\right)$ \\
headache & 39.6 & 58.9 & 92 & 84.5 & 30 \\
nausea and vomiting & 49.1 & 64.1 & - & - & 88 \\
sweating & 17.0 & 64.1 & - & - & - \\
abdominal pain & 30.2 & 57.4 & - & 51.0 & - \\
arthralgia & - & 47.7 & 51 & - & - \\
cough & 20.8 & 24.9 & - & - & - \\
photophobia & - & 18.5 & - & - & - \\
epistaxia & - & 9.2 & 24 & 20.0 & 11 \\
jaundice & - & 4.4 & 23 & 10.0 & 0 \\
hematuria & - & 3.8 & - & - & - \\
petechiae & - & 2.8 & 43 & 34.0 & 54 \\
conjunctivitis & - & 2.6 & - & - & - \\
\hline
\end{tabular}

Data are presented as percent

there is a lot of circulating spirochetes and in addition in urine too, could be the cause of renal glomerular or tubulointerstitial disease (Linnemann et al., 1978).

In present study there was no Jarisch-Herxheimer (JHR) reaction but there was JHR in two other studies, $54.1 \%$ and $0.8 \%$ of patients (Dworkin et al., 1998; Masoumi Asl et al., 2009).

Leukocytosis is reported in several studies in TBRF as well as in the ours (Arshi et al., 2002). In present study, leukocytosis was observed in 43 patients (81.1\%), but in 9 patients it was normal (17\%) and only in one case $(1.9 \%)$ there was a decrease in leukocytes count. All 53 patients were assessed for ESR in our study. ESR in 33 cases (62.2\%) was between 10 and $100 \mathrm{~mm} / \mathrm{h}$ and 10 patients (18.9\%) had elevated ESR above $100 \mathrm{~mm} / \mathrm{h}$. In addition in 10 patients $(18.9 \%)$ it was in normal range. With the few exceptions, other studies and most textbooks do not talk about high ESR values in RF (Arshi et al., 2002). Although elevated ESR and positive CRP are not emphasized as indicators of TRBF; but in this study, the mentioned laboratory parameters with past history of recurrent fever was highly indicative of TRBF. So considering basic laboratory examination in favourable epidemiological contexts can guide the clinicians to work up for TRBF. Lumbar puncture in 19 patients (35.8\%) was carried out and all of them were reported normal.

There is a national surveillance system for TRBF in Iran and because of the instructions for TBRF management in this system; the hospitalization rate and mortality rate especially in paediatric population has been decreased. 
The research on ticks in Qazvin province, where present study has been carried out, revealed that Ornithodoros tholozani was infected with Borrelia persica (Aghighi et al., 2007).

This finding was in agreement with other research which showed Ornithodoros tholozani was infected with Borrelia persica and Ornithodoros erraticus with Borrelia microti (Masoumi Asl et al., 2009). Other study also confirmed that most cases of borrelia caused by B. persica were transmitted by 0 . tholozani (Moemenbellah-Fard et al., 2009).

Borrelia persica also was diagnosed in $16.7 \%$ of the soft tick Ornithodouros tholozani in study which was carried out by Barmaki et al. (2010) in Takistan district, one of the cities of Qazvin province which is located $35 \mathrm{~km}$ west of Qazvin. This increase in prevalence comparing the previous study with prevalence of $3.7 \%$ in the same province and same tick species could be due to difference in sensitivity of PCR technique and microscopic following xenodiagnosis (Aghighi et al., 2007; Rafinejad et al., 2011). On the other hand, the overall prevalence average in Iran and other countries is comparable to this prevalence. Although the rate to some extend is different in provinces of Iran and world. For example, this rate was 3.7\% in Hamadan (Vatandoost et al., 2003), 8.8\% in Qazvin (Aghighi et al., 2007), 36.6\% in Semnan (Nekoui et al., 1999), and 2-40\% in other parts of the world (Assous and Wilamowski, 2009). The rate differences in provinces and different part of the world could be due to species of tick, different way of sampling and laboratory method.

The limitation of this study was unavailability of PCR for diagnosis. We suggest further research on different aspect of tick-borne relapsing fever in children including epidemiology, clinical and laboratory findings. In addition travellers to the rural areas with high prevalence of the disease should be made aware of the risk of tick-borne relapsing fever and use of appropriate control measures. Communicable disease supervision including tick-borne relapsing fever should be pursued as well. So relapsing fever should be considered when patients who reside or have vacationed in north-west of Iran exhibit a recurring febrile illness.

Acknowledgements: This paper is the result of MD. thesis in School of Medicine, Qazvin University of Medical Sciences. We deeply thank the staff of the Center for Clinical Research at Qazvin Children Hospital affiliated to Qazvin University of Medical Sciences for their help in preparing this paper.

\section{References}

Abidov, Z. I., Vasil'eva, I. S., Rakhimov, N. R., Gutova, V. P., Parpiev, A. M. (1993) Tick-borne relapsing fever morbidity in Namangan. Med. Parazitol. (Mosk.) 1, 32-35. (in Russian)

Aghighi, Z., Assmar, M., Piazak, N., Javadian, E., Seyedi Rashti, M. A., Kia, E. B., Rassi, Y., Vatandoost, H. (2007) Distribution of soft ticks and their natural infection with Borrelia in a focus of relapsing fever in Iran. Iran. J. Arthropod. Borne Dis. 1, 14-18.

Ayazi P.; Mahyar A.; Oveisi S.; Esmailzadehha N.; Nooroozi S. 
Arshi, S. H., Majidpour, A., Sadeghi, H., Emdadi, D., Asmar, M., Derakhshan, M. H. (2002) Relapsing fever in Ardabil, a northwestern province of Iran. Arch. Iran. Med. 5, 141-145.

Assous, M. V., Wilamowski, A. (2009) Relapsing fever borreliosis in Eurasia - Forgotten, but certainly not gone! Clin. Microbiol. Infect. 15, 407-414.

Barbour, A. G. (1989) Antigenic Variation in Relapsing Fever Borrelia Species: Genetic Aspects. American Society for Microbiology, Washington, D.C.

Barbour, A. G., Hayes, S. (1986) Biology of Borrelia species. Microbiol Rev. 50, 381-400.

Barclay, A. J. G., Coulter, J. B. S. (1990) Tick-borne relapsing fever in central Tanzania. Trans. R. Soc. Trop. Med. Hyg. 84, 852-856.

Barmaki, A., Rafinejad, J., Vatandoost, H., Telmadarraiy, Z., Mohtarami, F., Leghaei, S. H., Oshaghi, M. A. (2010) Study on presence of Borrelia persica in soft ticks in western Iran. Iran. J. Arthropod. Borne Dis. 4, 19-25.

Borgnolo, G., Denku, B., Chiabrera, F., Hailu, B. (1993a) Louse-borne relapsing fever in Ethiopian children: a clinical study. Ann. Trop. Paediatr. 13, 165-171.

Borgnolo, G., Hailu, B., Ciancarelli, A., Almaviva, M., Woldemariam, T. (1993b) Louse-borne relapsing fever. A clinical and an epidemiological study of 389 patients in Asella Hospital, Ethiopia. Trop. Geogr. Med. 45, 66-69.

Brasseur, D. (1985) Tick-borne relapsing fever in a premature infant. Ann. Trop. Paediatr. 5, 161-162.

Brown, V., Larouze, B., Desve, G., Rousset, J. J., Thibon, M., Fourrier, A., Schwoebel, V. (1988) Clinical presentation of louse-borne relapsing fever among Ethiopian refugees in northern Somalia. Ann. Trop. Med. Parasitol. 82, 499-502.

Cook, G., Zumla, A. (2002) Manson's Tropical Diseases. W.B. Saunders, Philadelphia.

Dai, Q., Restrepo, B. I., Porcella, S. F., Raffel, S. J., Schwan, T. G., Barbour, A. G. (2006) Antigenic variation by Borrelia hermsii occurs through recombination between extragenic repetitive elements on linear plasmids. Mol. Microbiol. 60, 1329-1343.

Dworkin, M. S., Anderson, D. E. Jr., Schwan, T. G., Shoemaker, P. C., Banerjee, S. N., Kassen, B. O., Burgdorfer, W. (1998) Tick-borne relapsing fever in the northwestern United States and southwestern Canada. Clin. Infect. Dis. 26, 122-131.

Felsenfeld, O. (1971) Borrelia: Strains, Vectors, Human and Animal Borreliosis. Warren H. Green, St. Louis.

Ghavami, M. B., Asmar, M., Piazak, N. (2002) Epidemiology of tick borne relapsing fever in Zanjan city. J. Zanjan Univ. Med. Sci. 9, 22-27. (in Persian)

Goubau, P. F. (1984) Relapsing fevers. A review. Ann. Soc. Belg. Med. Trop. 64, 335-364.

Linnemann, C. C. Jr., Barber, L. C., Dine, M. S., Body, A. E. (1978) Tick-borne relapsing fever in the eastern United States. Am. J. Dis. Child. 132, 40-42.

Mahram, M., Ghavami, M. B. (2009) Congenital tick-borne relapsing fever: report of a case with transplacental transmission in the Islamic Republic of Iran. East. Mediterr. Health J. 15, 761-764.

Masoumi Asl, H., Goya, M. M., Vatandoost, H., Zahraei, S. M., Mafi, M., Asmar, M., Piazak, N., Aghighi, Z. (2009) The epidemiology of tick-borne relapsing fever in Iran during 1997-2006. Travel. Med. Infect. Dis. 7, 160-164.

Melkert, P.W., Stel, H.V. (1991) Neonatal Borrelia infections (relapsing fever): report of 5 cases and review of the literature. East Afr. Med. J. 68, 999-1005.

Moemenbellah-Fard, M. D., Benafshi, O., Rafinejad, J., Ashraf, H. (2009) Tick-borne relapsing fever in a new highland endemic focus of western Iran. Ann. Trop. Med. Parasitol. 103, 529-537.

Naddaf, S. R., Kishdehi, M., Siavashi, M. R. (2011) Comparison of PCR-based diagnosis with centrifuged-based enrichment method for detection of Borrelia persica in animal blood samples. Iran. J. Arthropod. Borne Dis. 5, 7-12. 
Nekoui, H., Assmar, M., Amirkhani, A., Pyazak, N. (1999) Distribution of ticks and their association with Borrelia in Semnan province. Iran. J. Public Health 28, 103-109.

Rafinejad, J., Choubdar, N., Oshaghi, M. A., Piazak, N., Satvat, T., Mohtarami, F., Barmaki, A. (2011) Detection of Borrelia persica infection in Ornithodoros tholozani using PCR targeting rrs gene and xenodiagnosis. Iran. J. Public Health 40, 138-145.

Raoult, D., Roux, V. (1999) The body louse as a vector of reemerging human diseases. Clin. Infect. Dis. 29, 888-911.

Rebaudet, S., Barola, S. (2006) Epidemiology of relapsing fever borreliosis in Europe. FEMS Immunol. Med. Microbiol. 48, 11-15.

Sonenshine, D. E. (1997) Biology of Ticks. Oxford University Press, New York.

Southern, P. M., Sanford, J. P. (1969) Relapsing fever. A clinical and microbiological review. Medicine (Baltimore) 48, 129-149.

Vatandoost, H., Ghaderi, A., Javadian, E., Zahir Nia, A. H., Rassi,Y., Piazak, N., Kia, E. B., Shaeghi, M., Telmodarreiy, Z., Abolhasani, M. (2003) Distribution of soft ticks and their infection with Borrelia in Hamadan province, Iran. Iran. J. Public Health 32, 22-24.

Yagupsky, P., Moses, S. (1985) Neonatal Borrelia species infection. Am. J. Dis. Child. 139, 74-76. 